\title{
A loving embrace
}

\author{
Christopher Byrne, MD*
}

Keywords: Pediatrics, resuscitation, physician wellness

I cannot remember the last time I showed up for a shift with no one to be seen. Time for Timmies.

"Does anyone want anything?"

I am moonlighting at the department I hope to work in and am trying to make new work friends.

"Can I get a medium coffee, two milks? Thank you."

There is nothing quite like the satisfaction of that first sip of coffee in the morning. I look at the tracking board.

"STILL no one to be seen!?"

Soon, it will be time to get to work.

"Can we get a physician to resus?"

$\mathrm{Oh}$-sooner than I expected. I am the resus doc today. An awkward speed walk to the resuscitation room ensues. When I arrive, there is no patient. Three nurses.

"Three-month-old. VSA [vital signs absent]. Five minutes out."

My heart sinks. This is about to be my first pediatric arrest as a physician. I phone a friend. Several friends.

"Can we get a second physician to resus?"

"CODE PINK, emergency department."

The room fills. It is quiet. Emergency medical services arrive. Cardiopulmonary resuscitation (CPR) is ongoing. Unexpectedly, it is still quiet.

"Hello. Can we transfer the patient over and then get the story?"

Previously healthy. Last seen well during feeding two hours ago. Mom found unresponsive approximately 20 minutes ago.

I look at this beautiful child. He has blond, curly hair. His colour looks good. I touch his leg, and he is warm.
"All right. Let's do a pulse and rhythm check."

I turn to the monitor. Asystole. A colleague performs a bedside ultrasound. No cardiac activity.

"Our priority is to secure the airway. Can we try to get an [intravenous] IV?"

The mouth will not open. It is a tough IV.

BUZZ. BUZZ.

Two IO [intraosseous] lines in. Succinylcholine. Epinephrine.

"The mouth is opening. We got the tube!"

There are pink, frothy secretions. Asystole. No cardiac activity.

"Does anyone know if mom is en route?"

The RT [respiratory therapist] student bursts into tears. I look down. There are white and blue pyjamas on the floor. I am not sure, but I think I see sailboats, too.

"Does anyone know if mom is en route?"

A nurse, who is pregnant, wipes her eyes. It is her turn for CPR.

"Does anyone have any other ideas? And where is mom!?"

In my head, the decision has been made. This will be the last round of CPR. Or will it?

"Time of death, 8:14 a.m."

It is quiet again. A nurse picks up the pyjamas and covers the child from the neck down with a blanket. A colleague grabs my shoulder.

"Great work. We did everything we could."

I pick up the chart. I look at the child. I exhale.

"Please, let me know when family arrives."

I leave resus. I do not know what to do. I escape to the washroom. I cry. I suppose it is time to see the next patient.

My coffee is cold.

"Hello. I understand you're having some back pain. How can we help?"

From *University of Toronto, Toronto, ON.

Correspondence to: Dr. Christopher Byrne, Department of Medicine, University of Toronto, 190 Elizabeth Street, R. Fraser Elliot Building, Rm 3-805, Toronto, M5G 2C4, ON; Email: cbyrne2014@gmail.com

(C) Canadian Association of Emergency Physicians

CJEM 2019;21(6):814-815

DOI 10.1017/cem.2019.372 
Back at it. My mind wanders. I wonder if the patient senses how I feel.

"Mom is here."

"My apologies, but I need to step out for a moment." I walk back toward resus. This time, slowly. I look at the child. Mom is waiting just down the hall in the quiet room.
"I'm so sorry."

In that moment, I imagine this must be as deep as sorrow can get.

"Would you like to see him?"

To resus, again. It ends with a loving embrace between a mother and her only child—an image I will not forget. 